\title{
Earth analogues for past and future life on Mars: isolation of perchlorate resistant halophiles from Big Soda Lake
}

\author{
Toshitaka Matsubara ${ }^{1,2,3}$, Kosuke Fujishima ${ }^{4,5}$, Chad W. Saltikov ${ }^{2}$, Satoshi Nakamura ${ }^{3}$ \\ and Lynn J. Rothschild ${ }^{2,6}$ \\ ${ }^{1}$ Advanced Studies Laboratories (ASL), University of California Santa Cruz - NASA Ames Research Center, Moffett \\ Field, CA 94035-1000, USA \\ ${ }^{2}$ Department of Microbiology and Environmental Toxicology, University of California, Santa Cruz, Santa Cruz, CA 95064, \\ USA \\ ${ }^{3}$ Department of Bioengineering, Tokyo Institute of Technology, Yokohama 226-8501, Japan \\ ${ }^{4}$ University Affiliated Research Center (UARC), NASA Ames Research Center, Moffett Field, CA 94035-1000, USA \\ ${ }^{5}$ Earth-Life Science Institute, Tokyo Institute of Technology, Tokyo 152-8551, Japan e-mail: kosuke.fujishima@nasa.gov \\ ${ }^{6}$ NASA Ames Research Center, Moffett Field, CA 94035-1000, USA e-mail: Lynn.J.Rothschild@nasa.gov
}

\begin{abstract}
The Martian regolith is known to contain a maximum of $0.5 \%(\mathrm{w} / \mathrm{v})$ perchlorate $\left(\mathrm{ClO}_{4}^{-}\right)$that is toxic for most living organisms. With such high concentrations of perchlorates on Mars, is there any possibility of life? Here, in order to search and identify potential organisms on Earth, which could survive the perchlorate levels on Mars, we have isolated four perchlorate resistant, halophilic/halotolerant bacterial species from Big Soda Lake (BSL) in Nevada, USA. The 16S ribosomal RNA sequences revealed that these halophiles belong to the genera Bacillus, Alkalibacillus and Halomonas. Growth curves were obtained using a saline medium with different concentrations of magnesium, sodium and/or calcium perchlorate salt to simulate the Martian eutectic brine water. All four species, BSL1-4, grew in high saline media in the presence of perchlorates. This is the first growth experiment using multiple perchlorate salts. BSL3 relative to Halomonas salifodinae showed high maximum growth (Optical Density) comparing with other isolates in the presence of $1 \%$ perchlorate salts. Also, BSL1 relative to Bacillus licheniformis survived in the presence of 5\% Na-perchlorate, but growth was slower in the absence of Na-perchlorate. The results revealed that these new model microbes are capable of tolerating the hypothesized hypersaline and perchlorate-rich Martian subsurface water environment. Perchlorate-resistant halophile would serve as a new model to understand the biochemistry that may occur on Mars.

Received 5 August 2016, accepted 10 October 2016, first published online 28 November 2016
\end{abstract}

Key words: Astrobiology, halophile, Mars, model organism, perchlorate resistance.

\section{Introduction}

The Martian surface is highly arid and contains a mineralogical component likely attributable to chloride salts (McLennan et al. 2005; Osterloo et al. 2008). Although there are terrestrial halophiles that could survive periods in the evaporites of Mars (Rothschild 1990), their slow growth would be of limited use for the colonization of Mars. In the last decade, 0.4-0.6 wt \% perchlorates were found at the Phoenix landing site (Green Valley, $68.2^{\circ} \mathrm{N}, 125.9^{\circ} \mathrm{W}$ ) on the Martian surface (Hecht et al. 2009; Kounaves et al. 2014) and similar abundance of the perchlorate anion was also reported from the Rocknest aeolian deposit $\left(4.590^{\circ} \mathrm{S}, 137.448^{\circ} \mathrm{E}\right)$ in the Gale Crater by the Mars Science Laboratory (MSL) rover (Leshin et al. 2013). The widespread presence of the element chlorine on the Marian surface (Keller et al. 2006) and the reanalysis of the Viking Lander 1 and 2 sites (Chryse Planitia, 22.7 $\mathrm{N}, 48.2^{\circ}$ $\mathrm{W}$ and Utopia Planitia, $48.3^{\circ} \mathrm{N}, 226.0^{\circ} \mathrm{W}$, respectively) (Navarro-González et al. 2010) suggest a global distribution of perchlorate on the Red Planet. Very recently, the seasonal flow of brine water on the Martian surface slope was reconfirmed through the detection of magnesium and sodium perchlorates by the Mars Reconnaissance Orbiter (Ojha et al. 2015). The perchlorate ion is kinetically stable and is very soluble in water leading to very low eutectic temperatures ranging from -74 to $-34^{\circ} \mathrm{C}$, depending on the surrounding cations (Marion et al. 2010). While terrestrial perchlorates are often found associated with nitrate salts, such as the white-coloured nitrate at the Atacama Desert in Chile (Ericksen 1981), Phoenix's TEGA experiment and MSL's SAM instrument both implied an existence of soil oxychlorine compounds in the form of magnesium or calcium perchlorate (Hecht et al. 2009; Glavin et al. 2013).

There are perchlorate-reducing bacteria (PRB) known that can utilize perchlorate $\left(\mathrm{ClO}_{4}^{-}\right)$and chlorate $\left(\mathrm{ClO}_{3}^{-}\right)$as terminal electron acceptors. The perchlorate and chlorate are reduced to chlorite $\left(\mathrm{ClO}_{2}^{-}\right)$by perchlorate reductase (pcr; EC 1.97.1.-), which is subsequently dismutated into molecular $\mathrm{O}_{2}$ and $\mathrm{Cl}^{-}$ by chlorite dismutase (EC 1.13.11.49) (Coates \& Achenbach 2004; Carlström et al. 2015a). The pcrA gene encodes the catalytic subunit of perchlorate reductase and thus the 
detection of this gene indicates that perchlorate reductase gene is there (Nozawa-Inoue et al. 2008).

The salt composition within brines is important to consider regarding the search for life in hypersaline environments. In the Burns formation at Meridiani Planum on Mars, the abundance of calcium and magnesium sulphate minerals detected by the Opportunity rover conservatively reconstructs to an ancient water activity of $a_{w}=0.78-0.86$ and possibly as low as $a_{w}=0.5$ (Tosca et al. 2008). This shows that water activity was very low even though water was present. This environment would probably have been uninhabitable except for halophilic or halotolerant microorganisms (Fendrihan et al. 2012). Very recently, Martian brines were simulated and suggested that are grouped into three types, Type I, II and III (Fox-Powell et al. 2016). Type I alkaline carbonate-chloride brines evolved a concentrated $\mathrm{K}-\mathrm{Na}-\mathrm{HCO}_{3}-\mathrm{Cl}$ composition, and Type II brines were $\mathrm{Mg}_{-} \mathrm{SO}_{4}$-Cl-dominated and Type III brines were $\mathrm{Fe}-\mathrm{Mg}-\mathrm{SO}_{4}-\mathrm{Cl}$, resulting in brines were extremely acidic. Type II and III brines include high levels of sulphates and Type I and II brines were each represented by one evaporation pathway, whereas two evaporation pathways were investigated for Type III brines to capture the compositional and physicochemical diversity possible in their evolution.

Some of PRB show fast-growth or growth in brine water, however there are no species that can do both of them simultaneously (Esclapez et al. 2014; Melnyk et al. 2014; Carlström et al. 2015b). Thus, finding a fast-growing perchlorate-resistant halophile would not only serve as a model for how an indigenous Martian microbe could survive, but it also would have potential as a new 'chassis' organism (a model organism experimentally developed for bioengineering) for synthetic biology applications on Mars that would simplify human exploration and eventual settlement (Menezes et al. 2015; Rothschild 2016). This report describes the isolation and characterization of perchlorate-resistant halophiles from Big Soda Lake (BSL) in Nevada, which will serve as such chassis organisms.

\section{Materials and Methods}

\section{Screening perchlorate-resistant microbes from BSL samples}

We collected small salt rocks and water samples from the shoreline of BSL (Zehr et al. 1987), a hypersaline alkaline lake in Nevada, USA (39 $31^{\prime} 29.9994^{\prime \prime},-118^{\circ} 52^{\prime} 44.4^{\prime \prime}$, Fig. 1). Samples were collected into a sterile containers and brought back to the laboratory where they were stored at $4^{\circ} \mathrm{C}$ until analyzed. To culture microbes from these samples, a simple saline medium (SSM) was used and prepared as follows: $100 \mathrm{~g}$ sodium chloride; $10 \mathrm{~g}$ yeast extract; $7.5 \mathrm{~g}$ casein, acid hydrolysate; $25 \mathrm{ml} 2 \mathrm{M}$ Tris- $\mathrm{HCl}$ (adjusted to $\mathrm{pH} 7.5$ ), $15 \mathrm{~g}$ agar (for solid media only), and miliQ water adjusted to a volume of $1 \mathrm{~L}$. A $50 \%(\mathrm{w} / \mathrm{v})(2.3 \mathrm{M})$ magnesium perchlorate (Sigma-Aldrich, St. Louis, MO) stock solution was prepared and filter sterilized with a $0.22 \mu \mathrm{m}$ pore size membrane. The solid SSM containing $0.5 \%(\mathrm{w} / \mathrm{v})$ magnesium perchlorate was prepared by adding $50 \%(\mathrm{w} / \mathrm{v})$ magnesium-perchlorate solution to autoclaved SSM containing 1.5\% (w/v) agar.
Small salt rocks and water from BSL were re-suspended into SSM and plated onto SSM agar plates with $0.5 \%(\mathrm{w} / \mathrm{v}) \mathrm{Mg}$ perchlorate. Colonies that formed after a $48 \mathrm{~h}$ incubation at $37^{\circ} \mathrm{C}$ in the dark were isolated by re-streaking onto SSM agar plates.

\section{Phylogenetic analysis}

The 16S ribosomal RNA (rRNA) gene was polymerase chain reaction (PCR) amplified from the isolates using a common universal bacterial primer pair: 8F (5'-AGAGTTTGATCCT GGCTCAG-3') and 1492R (5'-GGTTACCTTGTTACGA CTT-3') (Turner et al. 1999). PCR products were purified using a PCR clean up kit (Epoch Life Science, Inc., Sugar Land, TX) and sequenced three times to obtain reliable coverage. These sequences were used as a query to search for similar sequences registered in the Ribosome Database Project (RDP: https://rdp.cme.msu.edu/index.jsp) with given options (Strain: Type, Source: Isolates, Size: >1200, Quality: good, Taxonomy: Nomenclatural, KNN matches: 20) (Cole et al. 2014). Multiple sequence alignments were performed using Clustal Omega software and further manually curated by Seaview4 (Gouy et al. 2010). PhyML software implemented in Seaview4 was used to estimate the Maximum Likelihood (ML) phylogenetic tree with given options (GTR model; NNI tree search; and Bootstrap branch support with 100 replicates).

\section{Detection of pcrA gene}

The partial pcrA gene was PCR-amplified from isolates and Azospira suillum PS, a perchlorate reducing bacterium (positive control, gifted from Dr. Bywaters), using the pcrA gene detection primer pair: pcrA320F (5'-GCGCCCACCAC TACATGTAYGGNCC-3') and pcrA598R (5'-GGTGGTC GCCGTACCARTCRAA-3') (Nozawa-Inoue et al. 2008). PCR was done using with OneTaq ${ }^{\circledR}$ Quick-Load $^{\circledR}$ 2X Master Mix with Standard Buffer (New England Biolabs, Ipswich, MA) for TA cloning according to the manufacturer's instructions. PCR products were purified and cloned into the pYES2.1/V5-His-TOPO ${ }^{\circledR}$ vector (Invitrogen, Carlsbad, CA) for TA cloning. Constructed plasmids were transformed into Escherichia coli DH5a (New England Biolabs, Ipswich, MA) and plated on an LB plate containing 1.5\% agar with $100 \mu \mathrm{g} \mathrm{ml}^{-1}$ ampicillin (final concentration). Colonies were spread on an LB agar plate, cultivated over night at $37^{\circ} \mathrm{C}$ in the dark and then collected for plasmid extraction. Plasmids were extracted using the GenCatch ${ }^{\mathrm{TM}}$ Plus Plasmid DNA Miniprep Kit (Epoch Life Science, Inc., Sugar Land, TX). The inserted DNA sequences were read using the forward and reverse primers (the forward primer was in the pYES2.1/V5-His-TOPO ${ }^{\circledR}$ vector kit and the reverse primer was pcrA598R).

\section{Salt tolerance assay}

Different concentrations of salt media (compositions are the same as SSM except for sodium chloride, and these media contain $0,2.5,5,7.5,10,12.5,15,17.5$ and $20 \%$ sodium chloride instead of $10 \%$ sodium chloride) and LB medium containing $1,2.5,5$ and $7.5 \%$ sodium chloride (final conc.), were prepared for salt tolerance assays. Four isolates from BSL, Bacillus subtilis 1A976 (Bacillus Genetic Stock Center, Columbus, OH) 


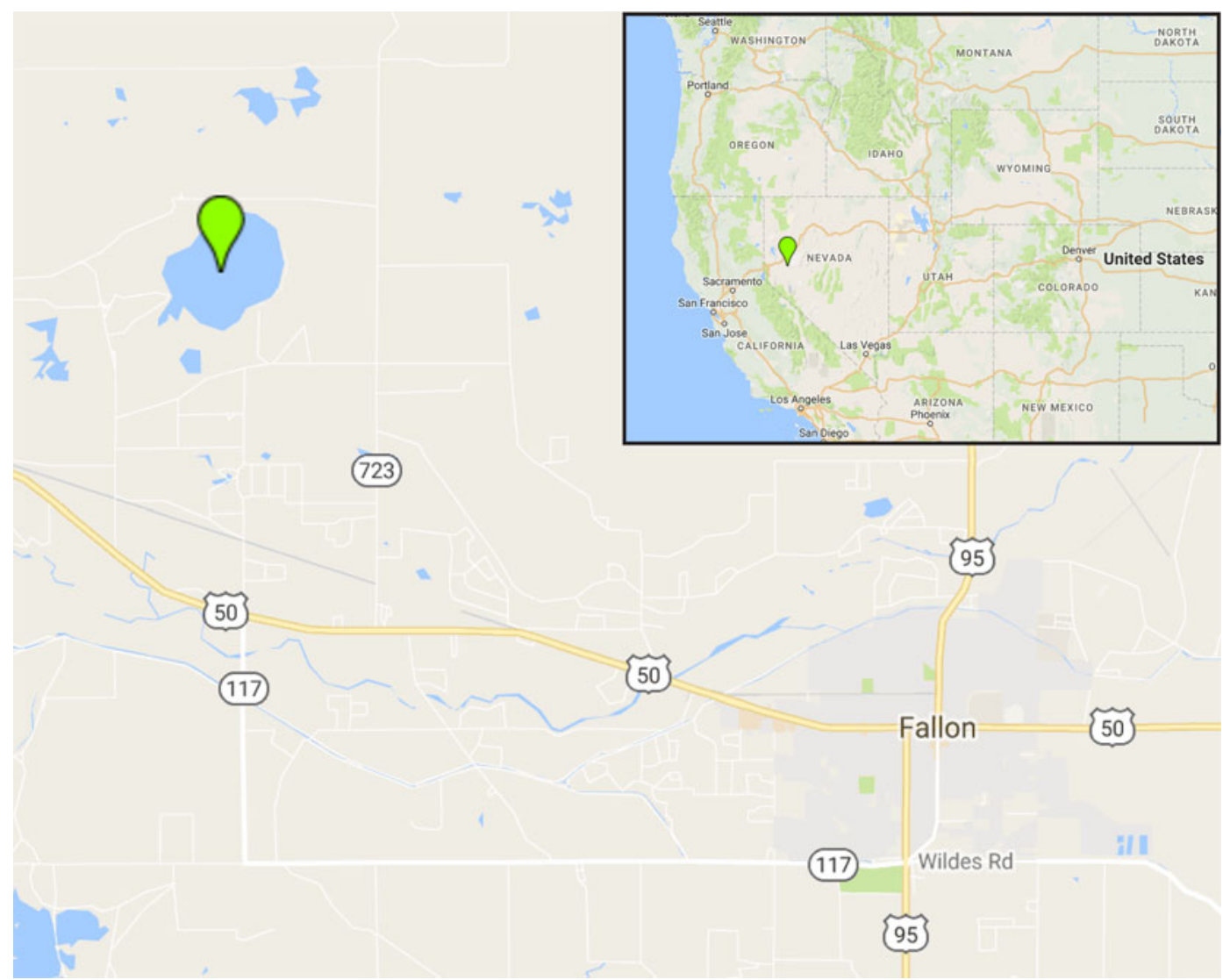

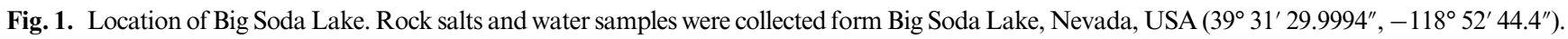
This map was created using the Google Maps.

and Escherichia coli DH5a were cultured overnight in SSM for four isolates and B. subtilis 1A976, and LB medium for E. coli DH5a (B. subtilis $1 \mathrm{~A} 976$ and $E$. coli DH5a were used for negative controls because they are relatively more sensitive to high salt concentrations compared with typical halophiles). For the assays, the cultures were inoculated with 1/200 volume of late-exponential cultures of the strains tested and incubated at $37^{\circ} \mathrm{C}$. The turbidity at OD $600 \mathrm{~nm}$ was measured each 20 min with shaking for periods up to $24 \mathrm{~h}$ using SpectraMax 384 Plus plate reader (Molecular Devices, Sunnyvale, CA, USA) with the included SoftMax ${ }^{\circledR}$ Pro (v5.3).

\section{Perchlorate resistance assay}

The four isolates were cultured overnight in SSM without the addition of perchlorate salt. Fifty per cent (w/v) (4.2 M) sodium perchlorate (Sigma-Aldrich, St. Louis, MO), 100\% $(\mathrm{w} / \mathrm{v})(4.2 \mathrm{M})$ calcium perchlorate (Sigma-Aldrich, St. Louis, MO) stock solution and 50\% (w/v) magnesium perchlorate stock solution were prepared. Then a $2 \mu$ pre-culture aliquot was added to $198 \mu \mathrm{l}$ of fresh SSM with one component perchlorate salt (magnesium, sodium or calcium perchlorate) or two components perchlorate salt of $0.5,1,1.5$ or $2 \%$. We also tested the growth in the presence of $5 \%$ sodium perchlorate to compare with the results in Oren et al. (2014). The concentration of two component perchlorate solution was prepared to add equal per cent (w/v) ratio of magnesium, sodium and/or calcium perchlorate stock solution. These mixtures were incubated at $37^{\circ} \mathrm{C}$. The turbidity (optical density (OD)) at $600 \mathrm{~nm}$ with shaking was measured every $20 \mathrm{~min}$ for up to $24 \mathrm{~h}$.

\section{Results}

Cellular characteristics of fast growing halophilic and halotolerant microorganisms from BSL

A total of four different isolates were identified, designated as BSL 1-4. As shown in Fig. 2, all isolates produced a circular colony except BSL1, which had a moist and shiny texture and BSL3, which had a slight yellow colour. Microscopic morphology of the BSL isolates revealed similar rod-shaped structures but with varying diameter and length (BSL1: $0.9 \times 4.3 \mu \mathrm{m}, \quad$ BSL2: $1.3 \times 5.0 \mu \mathrm{m}, \quad$ BSL3: $0.93 \times 2.9 \mu \mathrm{m}$, BSL4: $0.46 \times 5.0 \mu \mathrm{m}$, Fig. 2 ).

\section{Phylogenetic analysis and detection of pcrA gene of isolates}

The 16S rRNA sequences from the isolates were used to determine the most similar registered in the Ribosomal Database Project (RDP) database. This analysis revealed that BSL1, 2 and 4 belong to the order Bacillales within the phylum Firmicutes, while BSL3 belongs to the genus Halomonas within the phylum Proteobacteria. In order to estimate the 


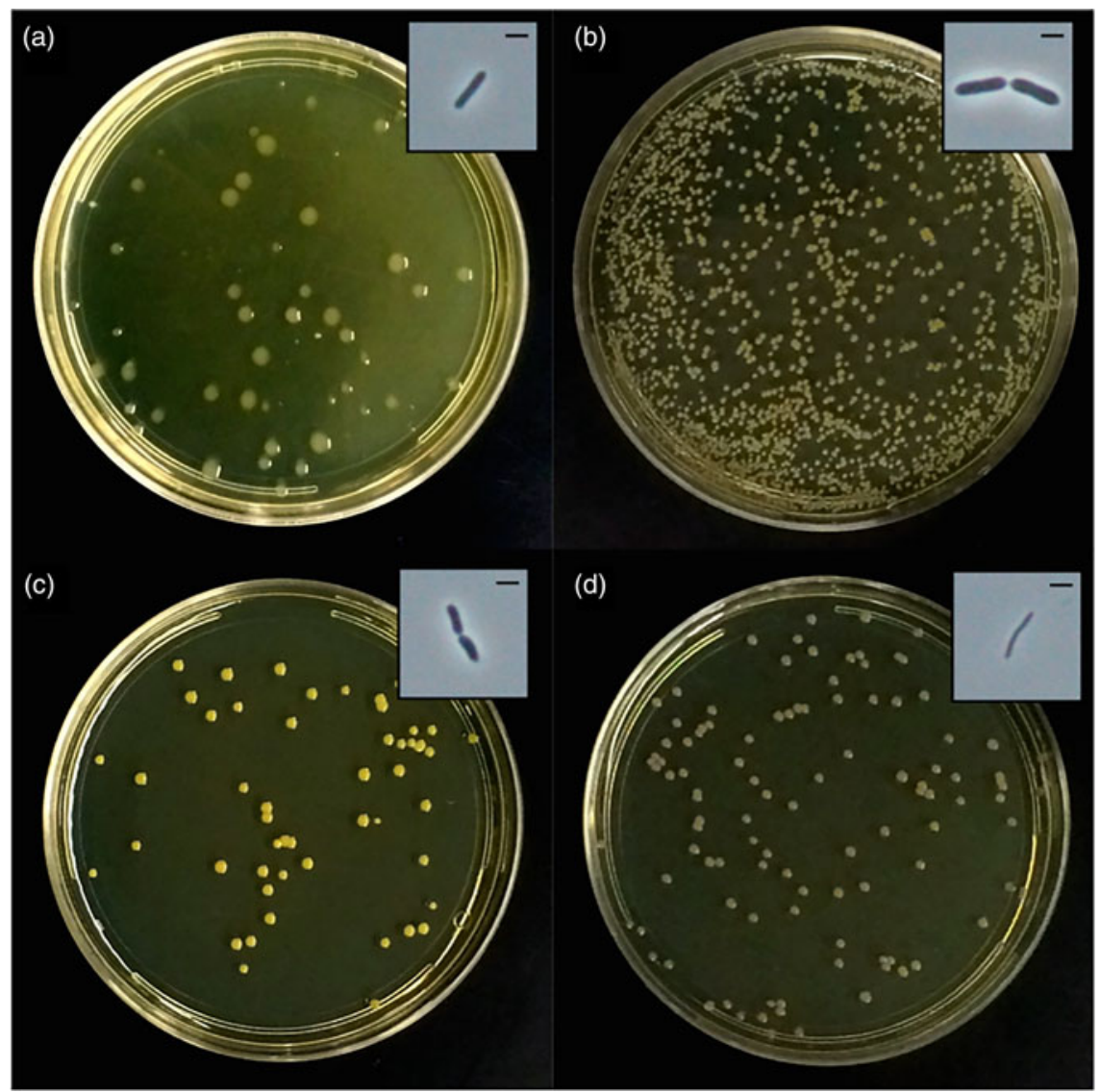

Fig. 2. Colony morphology and phase-contrast micrographs of perchlorate-resistant halotolerant or halophilic organisms isolated from Big Soda Lake. The four isolated cells named as Big Soda Lake (BSL) 1, 2, 3 and 4 are plated on SSM agar plate and cultured for 24 h. a, BSL1; b, BSL2; c, BSL3; d, BSL4. Right upper boxes represent phase-contrast micrographs of single cell observed at 1000x magnification with a reference of $2 \mu \mathrm{m}$ black bar.

phylogenetic relationships with related species, multiple alignment files were constructed for BSL1, 2 and 4 (total 34 sequences) and BSL3 (total 22 sequences) and ML phylogenetic trees were constructed, respectively, using PhyML. As a result, BSL1 shared the same root with Bacillus licheniformis (identity: 99.2\%), BSL2 with Bacillus pseudofirmus (identity: 99.1\%), BSL3 with Halomonas salifodinae (identity: 99.1\%) and BSL4 with Alkalibacillus filiformis (identity: 99.1\%) (Fig. 3). The pcrA gene was not detected in our isolates, inserted in TOPO TA cloning vector, but it was detected in the A. suillum PS (positive control) used in this study.

\section{Effects of salt on growth}

Growth curves of BSL1, BSL2, BSL3 and BSL4 have shown different salt resistance to sodium chloride: BSL1: $0-10 \%$, BSL2: $2.5-10 \%$, BSL3: $2.5-17.5 \%$ and BSL4: $7.5-15 \%$ sodium, respectively (Fig. 4). Negative control strains, B. subtilis 1A976 were only capable of growing in SSM with up to $7.5 \%$ sodium chloride and E. coli DH5a in LB up to 5\% sodium chloride. Also, the maximum specific growth rate (increase in cell numbers per hour) of BSL1, BSL2, BSL3 and BSL4, B. subtilis
1A976 and E. coli DH5a corresponded to $1.60,1.77,1.91$, $0.422,1.39$ and 1.71 in the presence of $2.5,5.0,5.0,10,2.5$ and $0 \%$ sodium chloride, respectively (Table 1). Cells of BSL1, BSL2, B. subtilis 1A976 and E. coli DH5a formed clumps during this assay and caused convexo-concave growth curves (Fig. 4(a), (b), (e) and (f)).

\section{Effects of perchlorates on growth}

Calcium, magnesium or sodium perchlorate salt was used for growth test of isolates and all isolate were grown in the presence of various perchlorate salts. BSL1 grew in the presence of $2 \%$ magnesium, sodium or calcium perchlorate (Fig. 5(a), (e) and (i)). BSL2 grew in the presence of 0.5 and $1.5 \%$ of magnesium and sodium perchlorates, respectively (Fig. 5(b), (f) and (j)). BSL3 grew in the presence of 1.5, 2 and $1.5 \%$ of magnesium, sodium and calcium perchlorates, respectively (Fig. 5(c), (g) and $(\mathrm{k}))$. BSL4 grew in the presence of 1.5 and $2 \%$ of magnesium and sodium perchlorates, respectively (Fig. 5(d), (h) and (1)). Perchlorate salts affected the growth of BSL1-4 and the growth rate became worse as perchlorate concentration increased. Calcium perchlorate salt reacted with SSM and generated white precipitates. This caused convexo-concave growth 
(a)

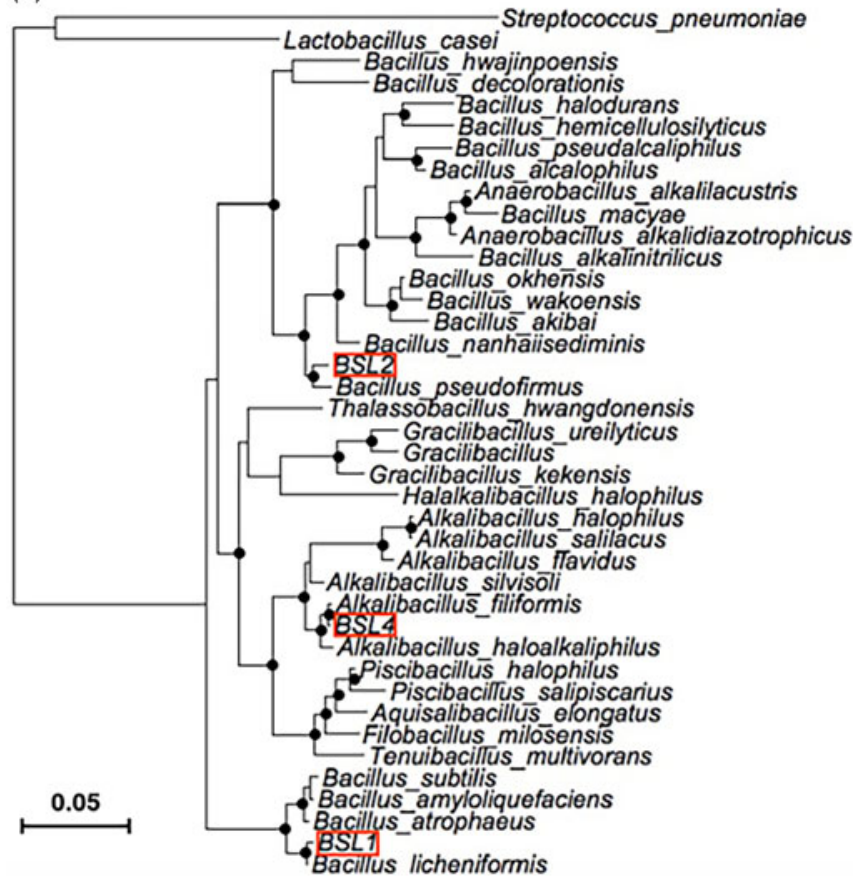

(b)

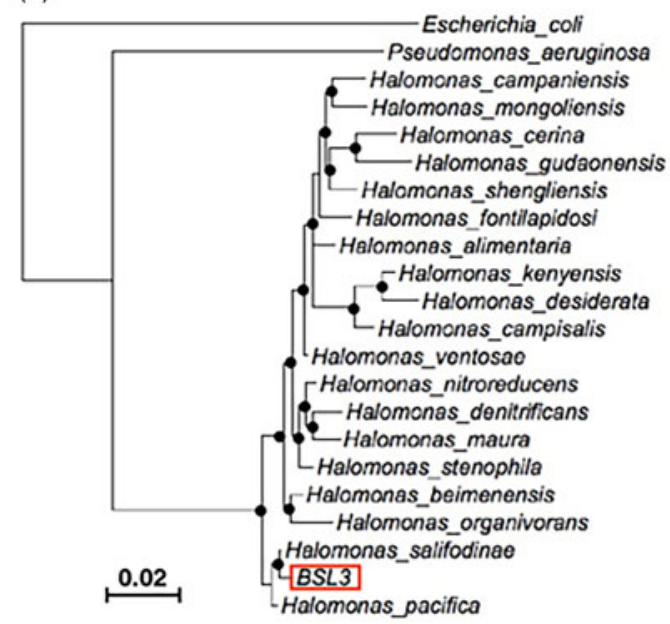

Fig. 3. Phylogenetic tree of isolated perchlorate resistant halophiles BSL1-4. Maximum likelihood 16S rRNA gene tree was generated by PhyML software implemented in Seaview4 (Gouy et al. 2010). Nodes with maximum likelihood support values above 80 using GTR model are indicated (black circle). (a) Phylogenetic tree of total 40 species from the order Bacillales including BSL1, BSL2 and BSL4 is illustrated with Lactobacillus casei and Streptococcus pneumoniae as an outgroup. (b) Phylogenetic tree of total 20 species from the genus Halomonas including BSL3 is illustrated with Escherichia coli and Pseudomonas aeruginosa as an outgroup.

(a)

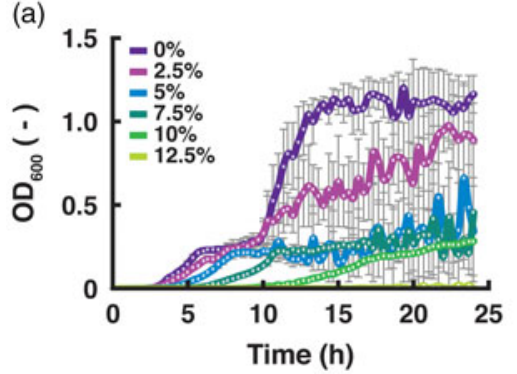

(d)

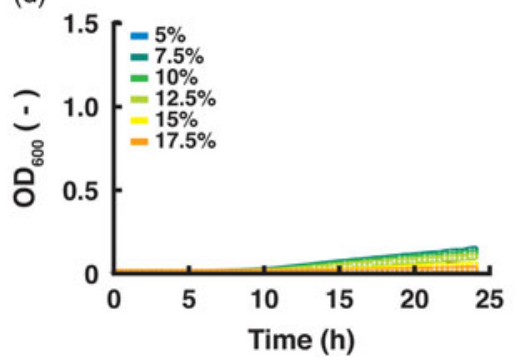

(b)

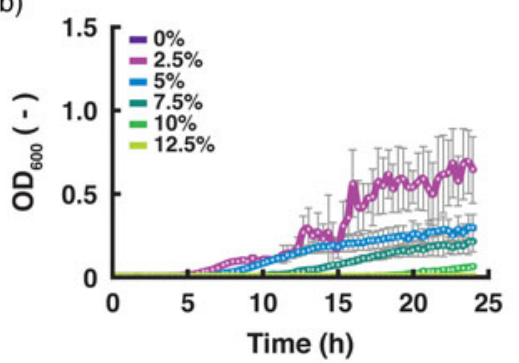

(e)

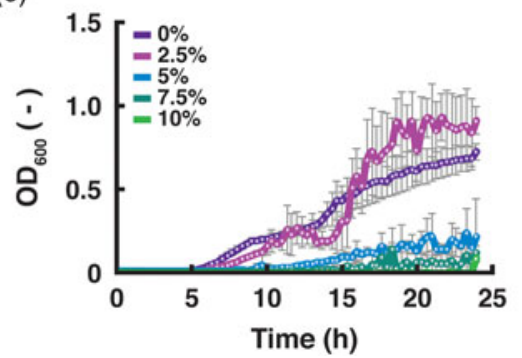

(c)

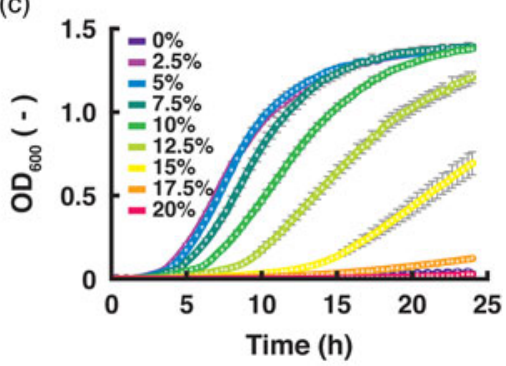

(f)

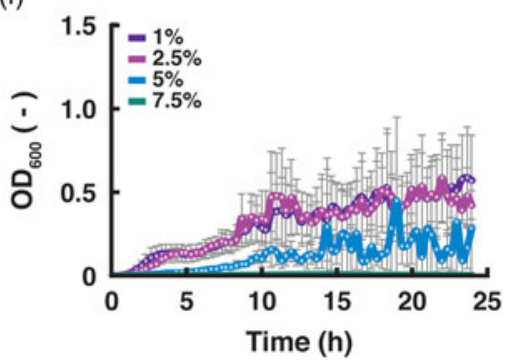

Fig. 4. Comparison of growth curves in the presence of different concentration of sodium chloride. Growth curves of BSL1-4, B. subtilis 1A976 and $E$. coli DH5a were measured for $24 \mathrm{~h}$ using 96 well plates with a SSM in the presence of 0 to $20 \%$ of sodium chloride (n $=9$ ). These graphs represent: a, BSL1; b, BSL2; c, BSL3; d, BSL4; e, B. subtilis 1A976; f, E. coli DH5a. Colors of symbol bars in graphs show differences in percentage of sodium chloride. Each point and error bar represents the average and standard deviation for 9 replicates. 
Table 1. Comparison of specific growth rates of BSL1-4 in the presence of different concentrations of sodium chloride.

\begin{tabular}{|c|c|c|c|c|c|c|c|c|c|}
\hline \multirow[b]{2}{*}{ Strain } & \multicolumn{9}{|c|}{ Salinity (\%) } \\
\hline & $0\left(1^{*}\right)$ & 2.5 & 5.0 & 7.5 & 10.0 & 12.5 & 15.0 & 17.5 & 20.0 \\
\hline $\mathrm{BSL}_{1}$ & 0.653 & 1.60 & 1.23 & 1.07 & 0.719 & - & - & - & - \\
\hline $\mathrm{BSL}_{2}$ & - & 1.05 & 1.77 & 1.52 & 0.759 & - & - & - & - \\
\hline $\mathrm{BSL}_{3}$ & - & 1.01 & 1.91 & 0.996 & 0.840 & 0.530 & 0.262 & 0.191 & 0.156 \\
\hline $\mathrm{BSL}_{4}$ & - & - & 0.0499 & 0.342 & 0.422 & 0.336 & 0.251 & & \\
\hline B. subtills 1A976 & 0.662 & 1.39 & 0.926 & 0.845 & 0.724 & 0.675 & - & - & - \\
\hline E. coli $\mathrm{DH} 5 \mathrm{a}$ & 1.71 & 1.53 & 0.562 & - & - & - & - & - & - \\
\hline
\end{tabular}

The unit of specific growth rates is $\mathrm{h}^{-1}$. LB medium was used for E. coli $\mathrm{DH} 5 \mathrm{a}$.

* LB medium including 1\% sodium chloride was used for $E$. coli DH5a.

(a)

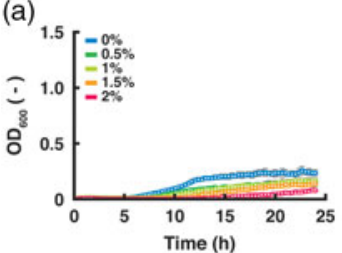

(e)

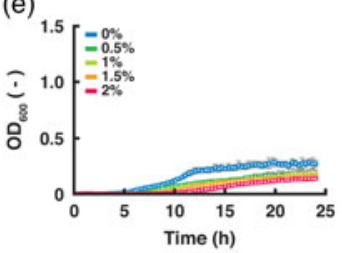

(i)

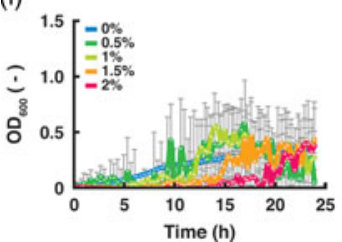

(b)

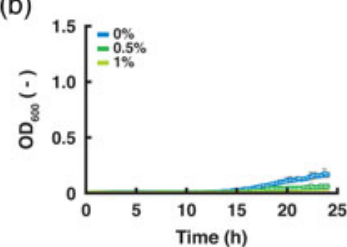

(f)

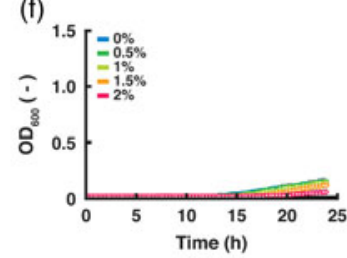

(j)

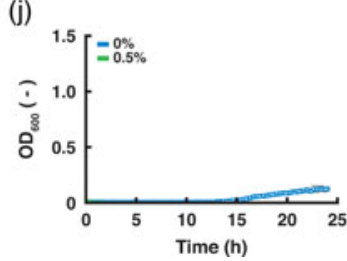

(c)

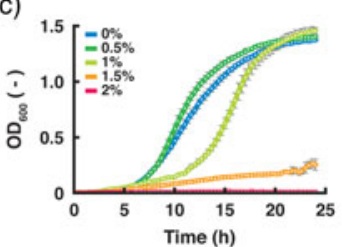

(g)

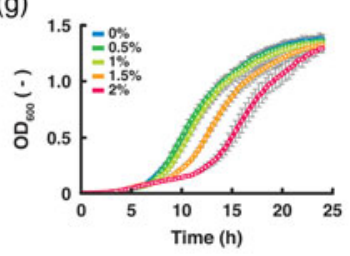

(k)

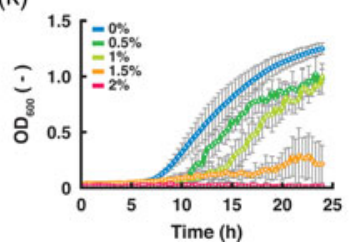

(d)

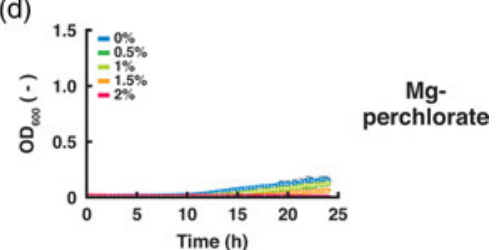

(h)

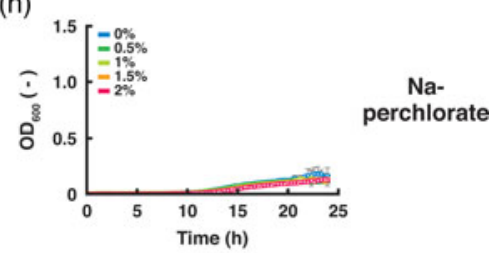

(I)

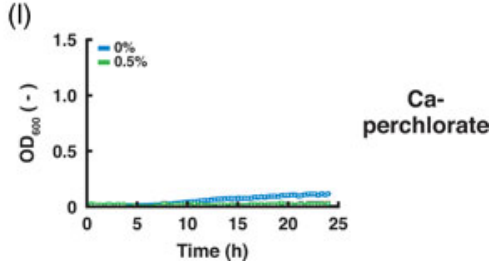

Fig. 5. Comparison of growth curve of BSL1-4 strains in SSM with varying perchlorate salt. Growth curves of BSL1-4 were measured for 24 h using 96 well plates with a SSM in the presence of 0 to $2 \%$ of magnesium, sodium and calcium perchlorate $(n=8)$. These graphs represent: a, e and i, BSL1; b, f and j, BSL2; c, g and k, BSL3; d, h and 1, BSL4. Each perchlorate was used: a-d, magnesium perchlorate; e- $h$, sodium perchlorate; i-1, calcium perchlorate. Colors of symbol bars in graphs show differences in percentage of each perchlorate. Each point and error bar represents the average and standard deviation for 8 replicates.

curves (Fig. 5(i) and (k)). Especially, BSL1 migrated with precipitates and caused wide range convexo-concave growth curves (Fig. 5(i)). Although, most of the growth curves in Fig. 5 were not very clear to calculate specific growth rate except for BSL3, as far as specific growth rate of BSL3 went almost same or value down with the increasing concentration of perchlorate salts (data not shown).

All isolate could be grown in the presence of two-component perchlorate salts (calcium, magnesium and/or sodium perchlorate salts) Moreover, the order of perchlorate tolerance were BSL3 > BSL1|BSL4 > BSL2 based on the growth curves comparisons (graphs of $0 \%$ and other percentage in Fig. 6). The results showed that the four BSL strains can be grown at least in the presence of $0.5 \%$ mixed magnesium, sodium and/or calcium perchlorate (Fig. 6). Growth curves in the presence of two-component perchlorate salt mixtures including calcium perchlorate were disposed to be convexo-concave growth curves (Fig. 6(e)-(1)) and this was prominently visible in BSL1 (Fig. 6(e) and (i)).

\section{Discussion}

The Martian surface is known to be highly arid and contains a mineralogical component likely attributable to chloride salts at a global scale (McLennan et al. 2005; Osterloo et al. 2008). The four isolates from BSL (BSL1, BSL2, BSL3 and BSL4) exhibited different growth rates in the presence of $0-20 \%$ sodium chloride (Fig. 4). According to the calculated specific growth rates and doubling times (Tables 1 and 2), BSL3 showed the slowest but constant growth even in $20 \%$ sodium chloride, which is approximately six times the concentration of the Earth's oceans. On the other hand, B. subtilis 1 A976 and E. 
(a)

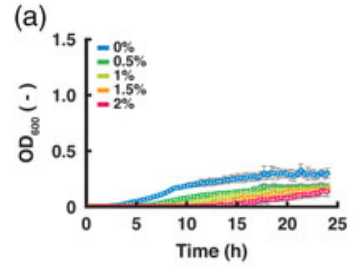

(e)

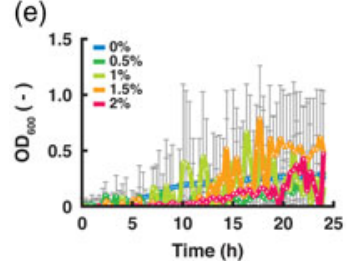

(i)

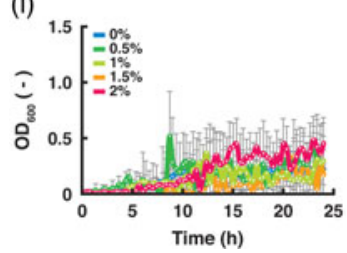

(b)

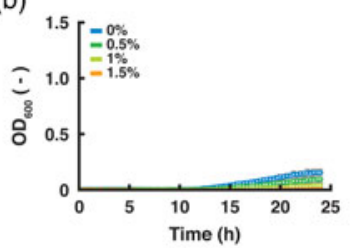

(f)

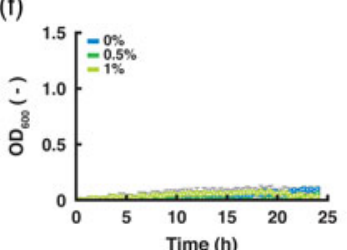

(j)

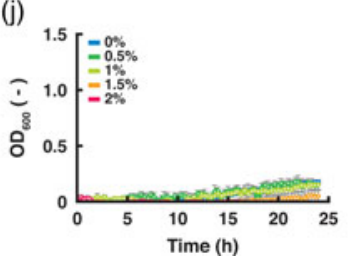

(c)

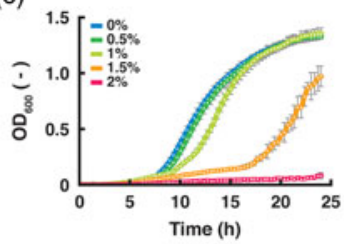

(g)

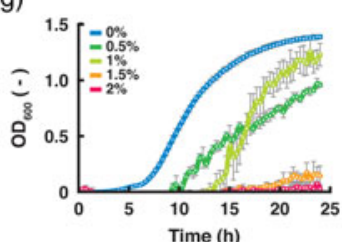

(k)

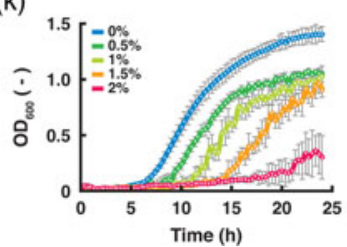

(d)

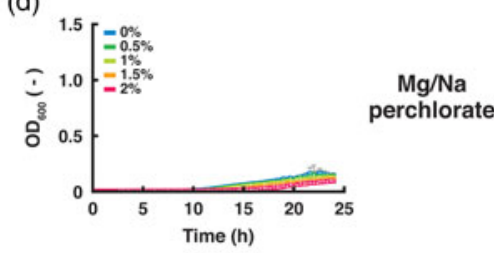

(h)

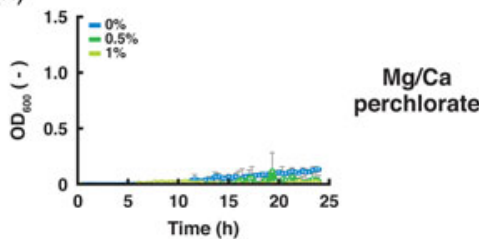

(I)

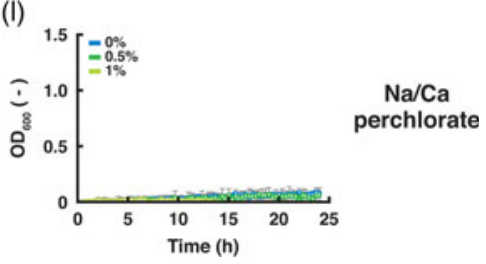

Fig. 6. Comparison of growth curve of BSL1-4 strains in SSM with varying two-component perchlorate salt mixtures. Growth curves of BSL1-4 were measured for $24 \mathrm{~h}$ using 96 well plates with a SSM in the presence of 0 to $2 \%$ mixture of magnesium, sodium and/or calcium perchlorates $(\mathrm{n}=8)$. These graphs represent: a, e and i, BSL1; b, f and j, BSL2; c, g and k, BSL3; d, h and 1, BSL4. Each mixed perchlorate was used: a-d, magnesium and sodium perchlorates; e-h, magnesium and calcium perchlorates; $\mathrm{i}-1$, sodium and calcium perchlorates. Colors of symbol bars in graphs show differences in percentage of each perchlorate. Each point and error bar represents the average and standard deviation for 8 replicates.

coli DH5a, which are typically used as 'chassis' for genetic manipulation, could not survive above the threshold of $10 \%$ sodium chloride. Specific growth rate of BSL3 and B. subtilis 1 A976 in the presence of $10 \%$ sodium chloride was $0.840 \mathrm{~h}^{-1}$ (doubling time was $49.5 \mathrm{~min}$ ) and $0.724 \mathrm{~h}^{-1}$ (doubling time was $57.4 \mathrm{~min}$ ), respectively, and there is not great difference between them. However, their maximum growth ODs were totally different. Although specific growth rate is a useful indicator for deciding how fast a culture can grow, other parameters should also be considered. It is necessary to consider using data of maximum growth OD, length of lag phase, addition to specific growth rates. Together with the recent observation of seasonal brine water flow on Martian surface, our isolates have more potential than B. subtilis $1 \mathrm{~A} 976$ and E. coli DH5a to be model organisms for growing under Mars-like conditions, which would utilize the high-saline Martian subsurface water as a source for culture media. Also, the Martian brines were grouped into three types: Type I brines, concentrated $\mathrm{K}-\mathrm{Na}-\mathrm{HCO}_{3}-\mathrm{Cl}$; Type II brines, concentrated $\mathrm{Mg}-\mathrm{SO}_{4}-\mathrm{Cl}$; Type III brines, concentrated $\mathrm{Fe}-\mathrm{Mg}-\mathrm{SO}_{4}-\mathrm{Cl}$. Type II and III brines include high levels of sulphates (Fox-Powell et al. 2016). While our four isolates have shown survivability in the presence of $2 \% \mathrm{MgSO}_{4}$ (data not shown), this relatively new knowledge on the presence of sulphate rich brine water may provide further restriction on the redox cycle and overall growth of the model organisms.

While our isolates expressed wide range of sodium chloride tolerance, our motivation is to find a new model organisms that can survive in the presence of perchlorate, given the evidence of magnesium, sodium and calcium perchlorates existing on the Martian surface as well as the recurring slope lineae, where subsurface water could be still present (Hecht et al. 2009; Glavin et al. 2013; Martín-Torres et al. 2015; Ojha et al. 2015). Thus, perchlorate tolerance is critical to the utilization of brine water as a resource on Mars. Here our isolates were screened in the presence of $0.5 \%$ magnesium perchlorate and we have shown that these isolates have the ability to tolerate various perchlorate salts including magnesium, sodium and calcium perchlorates (Fig. 5). So far several members of the archaeal family belonging to Halobacteriaceae and the bacterium Halomonas have been shown to survive in the presence of 5\% sodium perchlorate (Oren et al. 2014). Here we tested our four isolates under the same perchlorate concentration in SSM and found that BSL1 was the only strain that survived under this conditions (Fig. 7). The only inconsistency with Oren et al. (2014) was that survivability of Halomonas species but this could be due to the difference in incubator (96-well plate versus shaking flask) and nutrient level (SSM versus rich and defined media).

All of the isolates survived in the presence of $2 \%$ sodium perchlorate, while growth rate decreased or was strongly inhibited in the presence of magnesium and calcium perchlorates (Fig. 5). Magnesium and calcium perchlorates had more negative effects than sodium perchlorate on bacterial growth. According to the molarity of perchlorate ions in the growth medium, 2\% sodium perchlorate (160 mM perchlorate ions) contains more perchlorate ions than that of $1.5 \%$ magnesium or calcium perchlorate (138 or $126 \mathrm{mM}$ perchlorate ions), 
Table 2. Comparison of doubling time of BSL1-4 in the presence of different concentration of sodium chloride.

\begin{tabular}{lccccccccc}
\hline & \multicolumn{2}{l}{ Salinity (\%) } \\
\cline { 2 - 8 } Strain & $0\left(1^{*}\right)$ & 2.5 & 5.0 & 7.5 & 10.0 & 12.5 & 15.0 & 17.5 \\
\hline BSL $_{1}$ & 63.7 & 26.0 & 33.8 & 38.8 & 57.9 & - & - & - \\
BSL $_{2}$ & - & 39.5 & 23.5 & 27.4 & 54.8 & - & - & - \\
BSL $_{3}$ & - & 41.2 & 21.8 & 41.8 & 49.5 & 78.4 & 158.7 & 218.2 & 26.17 \\
BSL $_{4}$ & - & - & 833.0 & 121.7 & 98.7 & 123.7 & 166.0 & - \\
B. subtills 1 A976 & 62.8 & 29.8 & 44.9 & 49.2 & 57.4 & 61.6 & - & - \\
E. coli DH5a & 24.3 & 27.2 & 74.0 & - & - & - & - & - \\
\hline
\end{tabular}

The unit of doubling time is minute. LB medium was used for $E$. coli DH5a.

* LB medium including $1 \%$ sodium chloride was used for $E$. coli DH5a.
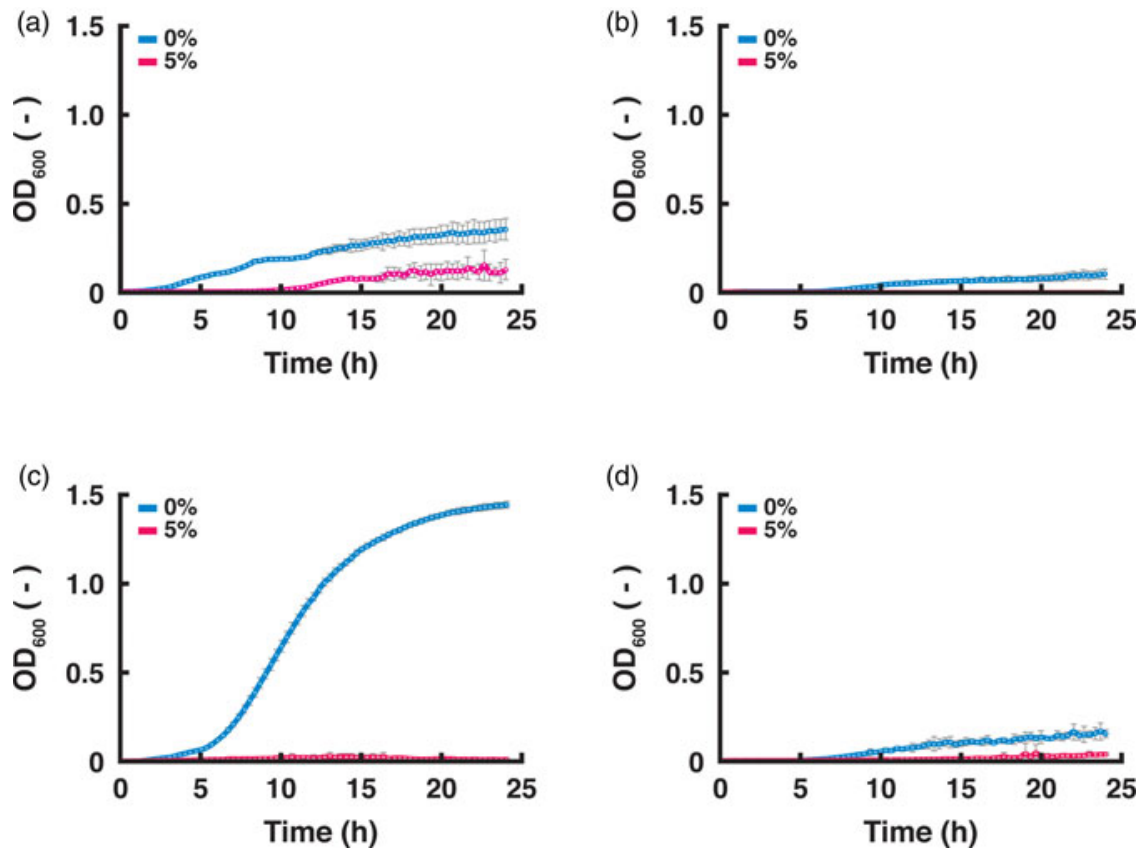

Fig. 7. Comparison of growth curves of BSL1-4 strains in SSM with 5\% sodium perchlorate. Growth curves of BSL1-4 were measured for 24 h using 96 well plates with SSM with no perchlorate (blue) and 5\% of sodium perchlorate (red) (n=9). a, BSL1; b, BSL2; c, BSL3; d, BSL4. Each point and error bar represents the average and standard deviation for 9 replicates.

suggesting that the negative effect on bacterial growth is caused by not only the perchlorate ions, but also due to the type of coexisting metal ions.

Since several perchlorate salts are considered to coexist on Mars (Hecht et al. 2009; Glavin et al. 2013; Kounaves et al. 2014), we tested the growth rate under more realistic Martian environmental conditions in which two of the three perchlorate salt components (sodium, magnesium and calcium) were added. The combination of different salts had no synergistic effect on the overall survivability of the four isolates, but rather showed intermediate growth behaviours of the results observed under individual perchlorate salts (Figs. 5 and 6). However, calcium perchlorate seemed to cause a precipitation in the culture medium making the OD reading difficult especially for BSL1.

Perchlorate reducing bacteria typically have the $p c r A$ gene. Although we were unable to detect PRB genes in our perchlorate tolerant $\mathrm{BSL}$ strains, it suggests that an alternative pathway may exist to explain their resistance. Assuming that perchlorate salt is present globally on Martian soil, inhalation of Martian dust, especially dust particles $<5 \mu \mathrm{m}$ in size is a particular concern, given the fact that particles of this size cannot be expelled by lung mucus (Davila et al. 2013). The primary toxicity issue of perchlorates for humans is associated with the disruption of iodide uptake in the thyroid gland due to its similarity in ionic radius to iodide. The stability of perchlorates as a strong oxidant allows perchlorate salts to remain in the environment. Reverse osmosis or other engineering technology will likely be employed to obtain pure water for various usages on Mars. However, such processes require pressure pumps, filter and mechanical parts that may need periodic servicing and replacement, which adds to the overall cost of transporting those materials to Mars. Conversely, using a microbe that can grow under Martian condition is favoured in terms of reducing the net weight and volume. Perchlorates are also hard to 


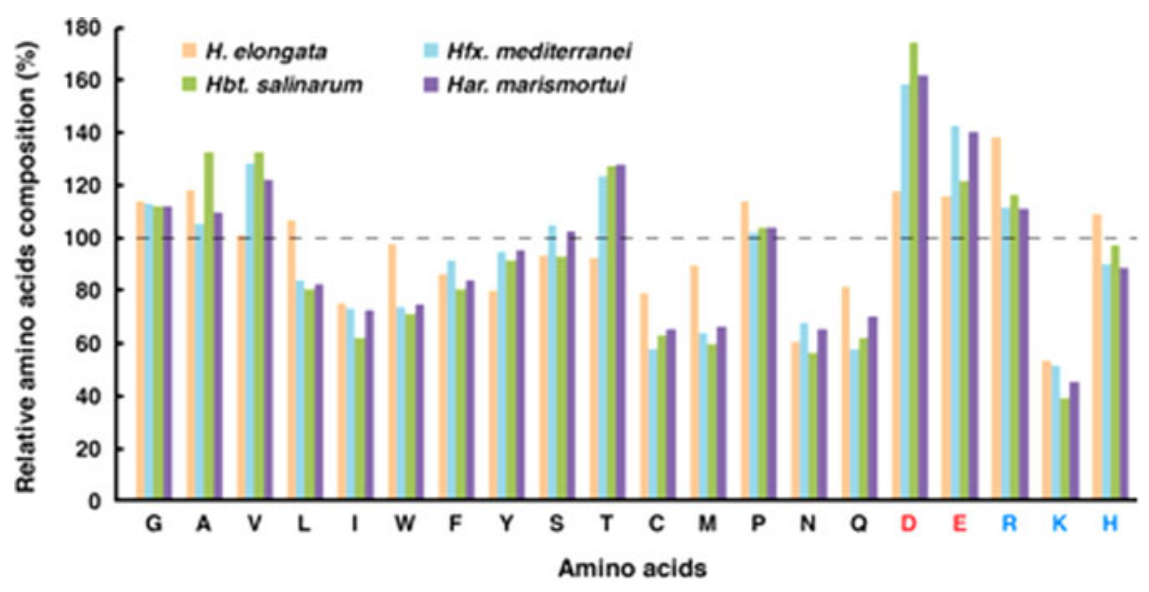

Fig. 8. Amino acids composition of perchlorate tolerant halophiles relative to E. coli. Amino acids compositions of four halophilic microbes calculated relative to $E$. coli $\mathrm{K} 12$ based on their proteome data obtained from UniProt (http://www.uniprot.org/). Dash line indicates the compositions of 20 amino acids in E. coli $\mathrm{K} 12$ as a $100 \%$ baseline. Red and blue amino acid characters represent acidic and basic amino acid, respectively. The four known perchlorate tolerant halophiles shown as bar graph are Halomonas elongate ATCC 33173 (orange), Haloferax mediterranei ATCC 33500 (blue), Halobacterium salinarum strain ATCC 29341 (green) and Haloarcula marismortui ATCC 43049 (purple) (Oren et al. 2014).

treat by chemical reduction, while biological reactions can effectively reduce and degrade perchlorate under anoxic conditions releasing oxygen (Srinivasan \& Viraraghavan 2009). Thus, biodegradation may become the key process to detoxify perchlorates and produce oxygen for sustainable human exploration on Mars. PRB can utilize perchlorate $\left(\mathrm{ClO}_{4}^{-}\right)$ and chlorate $\left(\mathrm{ClO}_{3}^{-}\right)$as terminal electron acceptors and reduce perchlorate and chlorate to chlorite $\left(\mathrm{ClO}_{2}^{-}\right)$. By utilizing the perchlorate reductase enzyme (Pcr) present in PRB, chlorite is subsequently dismutated into molecular $\mathrm{O}_{2}$ and $\mathrm{Cl}^{-}$by chlorite dismutase (Coates \& Achenbach 2004; Carlström et al. 2015a). Chlorite dismutation is the only chemotrophic microbial metabolism shown to produce significant amounts of free $\mathrm{O}_{2}$ (Coates \& Achenbach 2004). While BSL1, BSL2, BSL3 and BSL4 may not possess known perchlorate reductase and chlorate dismutase genes (data not shown), they could still survive in the presence of perchlorate salts, thus serving as a potential model organism for bioengineering perchlorate reduction for oxygen bioproduction on Mars, alternative to photosynthesis. So far no PRB have been discovered to show a fast growth reaching high $\mathrm{OD}\left(\mathrm{OD}_{600}=1.0\right)$ within $24 \mathrm{~h}$ in brine water (Esclapez et al. 2014; Melnyk et al. 2014; Carlström et al. 2015b). Our findings of a fast-growing perchlorate-resistant halophile would serve as a model for how an indigenous Martian microbe could be surviving underground, as well as its application as a new 'chassis' organism for synthetic biology applications on Mars (Menezes et al. 2015; Rothschild 2016).

All four isolates (BSL1-4) should be capable for genetic engineering and suggested to grow under anaerobic conditions. From the point of view of genetic manipulation, B. licheniformis and B. pseudofirmus are generally known to be Grampositive, facultative anaerobic, spore-forming bacteria. As with $B$. subtilis and B. licheniformis relative to BSL1 are nontoxic host organisms used for the production of harmless industrial products such as industrial enzymes, biochemicals and consumer products, and for producing extracellular enzymes (de Boer et al. 1994; Rey et al. 2004). Also, B. pseudofirmus relative to BSL2 is known as a facultative alkaliphile and a model organism for studies of bioenergetics at high $\mathrm{pH}$ (Janto et al. 2011). Also, many Bacillus species can be grown under the anaerobic condition. $H$. salifodinae, a relative to BSL3, is a Gram-negative, aerobic, halophilic bacterium (Wang et al. 2008). The genus Halomonas was recently used for the production of polyhydroxyalkanoates bioplastics under sea water conditions (Tan et al. 2014; Yue et al. 2014). These recombinant Halomonas species are transformed using plasmids through conjugation, which is so far the only known procedure for genetic engineering of Halomonas species. Also, some of the Halomonas species are reported to grow under anaerobic condition (Vreeland et al. 1980; Maskow \& Babel 2003). An A. filiformis relative to BSL4 is a halo-alkaliphilic, Gram-positive, aerobic bacterium and the genus Alkalibacillus is a novel genus based on a reclassification of Bacillus (Jeon et al. 2005; Romano et al. 2005) and so far no genetic engineering application has been reported.

While all four isolates survived various perchlorate concentration, BSL3 showed the fastest growth $(24 \mathrm{~h})$ to reach the OD level up to 1.0 even at $1 \% \mathrm{Mg} / \mathrm{Ca}$ perchlorate salt concentration (Fig. 6). This result suggests that this microorganism could be used for utilizing Martian subsurface brine water for human Mars exploration or in an arid and perchlorate-rich environment on Earth where current model organisms such as $B$. subtilis and $E$. coli could not survive. Therefore, if we construct perchlorate degradable fast-growing microorganisms utilizing isolates by synthetic biological methods, they would be chassis for perchlorate degradation, which means that without any delay there will be fast-growth in the presence of perchlorate salt and perchlorate will be efficiently degraded and this would be very helpful and play an important role for human exploration of Mars. Halomonas relative to BSL3 is halophilic 
bacteria. BSL3 may become capable of reducing the perchlorate only after it has been genetically engineered. Also, there are a lot of useful enzymes and products from halophilic archaea such as glycosyl hydrolases, lipases, esterases, proteases, biopolymers and surfactants (LitchWeld 2011). It would be very useful if these products were produced from fast-growing halophiles.

From the standpoint of potential life on present Mars, halophilic/halotolerant and perchlorate resistant feature will be important to survive in subsurface saline environment. If Martian microbe relies on halophilic proteins to convey chemical reactions, the feature that we see in terrestrial halophilic protein could be applicable. For example, halophilic proteins represent a large increase in acidic amino acids to compete with salt for water binding capability and use of relatively smaller hydrophobic amino acids leading to smaller hydrophobic core (Reed et al. 2013). Also increase in arginine to lysine ratio is another feature seen during the haloadaptation, due to the fact that arginine can bind more water molecules than lysine (Pieper et al. 1998) resulting in the reduction of hydrophobic surface (Britton et al. 2006). We performed amino acid composition analysis on the four known perchlorate tolerant halophiles (Fig. 8) and found the same trend described above. Therefore this feature could be useful for distinguishing extant Martian biosphere from terrestrial biological contaminants when using amino acid profile as a biomarker for life detection on Mars.

\section{Acknowledgements}

We would like to thank the NASA Ames Research Center Director's Fund and Synthetic Biology Program at NASA Headquarters for support. We thank Dr. Kathryn Bywaters (NASA Ames Research Center, USRA) for kindly providing A. suillum for pcrA gene detection. This work was partly supported by JSPS KAKENHI (14J10566) to T. M. Finally, we would like to thank S. Pete Worden for encouragement to explore the use of synthetic biology in Mars exploration and the issue of perchlorates on Mars in particular. As for KF, this publication was supported by the ELSI Origins Network (EON), through a grant from the John Templeton Foundation.

\section{References}

Britton, K.L., Baker, P.J., Fisher, M., Ruzheinikov, S., Gilmour, D.J., Bonete, M.J., Ferrer, J., Pire, C., Esclapez, J. \& Rice, D.W. (2006). Analysis of protein solvent interactions in glucose dehydrogenase from the extreme halophile Haloferax mediterranei. Proc. Natl. Acad. Sci. USA. 103, 4846-4851.

Carlström, C.I., Loutey, D., Bauer, S., Clark, I.C., Rohde, R.A., Iavarone, A.T., Lucas, L. \& Coates, J.D. (2015a). (Per)chlorate-reducing bacteria can utilize aerobic and anaerobic pathways of aromatic degradation with (Per)chlorate as an electron acceptor. mBio 6, e02287-e022814.

Carlström, C.I., Loutey, D.E., Wang, O., Engelbrektson, A., Clark, I., Lucas, L.N., Somasekhar, P.Y. \& Coates, J.D. (2015b). Phenotypic and genotypic description of Sedimenticola selenatireducens strain CUZ, a marine (per) chlorate-respiring gammaproteobacterium, and its close relative the chlorate-respiring Sedimenticola strain NSS. Appl. Environ. Microbiol. 81, 2717-2726.

Coates, J.D. \& Achenbach, L.A. (2004). Microbial perchlorate reduction: rocket-fueled metabolism. Nat. Rev. Microbiol. 2, 569-580.

Cole, J.R., Wang, Q., Fish, J.A., Chai, B., McGarrell, D.M., Sun, Y., Brown, C.T., Porras-Alfaro, A., Kuske, C.R. \& Tiedje, J.M. (2014). Ribosomal Database Project: data and tools for high throughput rRNA analysis. Nucl. Acid. Res. 42, D633-D642.

Davila, A.F., Willson, D., Coates, J.D. \& McKay, C.P. (2013). Perchlorate on Mars: a chemical hazard and a resource for humans. Int. J. Astrobiol. 12, 321-325.

de Boer, A.S., Priest, F. \& Diderichsen, B. (1994). On Industrial use of Bacillus licheniformis: a Review. App. Microbiol. Biotechnol. 40, 595-598.

Ericksen, G.E. (1981). Geology and origin of the Chilean nitrate deposits. Geol. Surv. Pro. Paper 1188.

Esclapez, J., Bravo-Barrales, G., Bautista, V., Pire, C., Camacho, M. \& Bonete, M.J. (2014). Effects of nitrogen sources on the nitrate assimilation in Haloferax mediterranei: growth kinetics and transcriptomic analysis. FEMS Microbiol. Lett. 350, 168-174.

Fendrihan, S., Dornmayr-Pfaffenhuemer, M., Gerbl, F.W., Holzinger, A., Grösbacher, M., Briza, P., Erler, A., Gruber, C., Plätzer, K. \& Stan-Lotter, H. (2012). Spherical particles of halophilic archaea correlate with exposure to low water activity-implications for microbial survival in fluid inclusions of ancient halite. Geobiology 10, $424-433$.

Fox-Powell, M.G., Hallsworth, J.E., Cousins, C.R. \& Cockell, C.S. (2016). Ionic strength is a barrier to the habitability of Mars. Astrobiolology 16, 427-442.

Glavin, D.P. et al. (2013). Evidence for perchlorates and the origin of chlorinated hydrocarbons detected by SAM at the Rocknest aeolian deposit in Gale Crater. J. Geophys. Res.: Planets 118, 1955-1973.

Gouy, M., Guindon, S. \& Gascuel, O. (2010). SeaView version 4: a multiplatform graphical user interface for sequence alignment and phylogenetic tree building. Mol. Biol. Evol. 27, 221-224.

Hecht, M.H. et al. (2009). Detection of perchlorate and the soluble chemistry of martian soil at the Phoenix lander site. Science 325, 64-67.

Janto, B. et al. (2011). Genome of alkaliphilic Bacillus pseudofirmus OF4 reveals adaptations that support the ability to grow in an external $\mathrm{pH}$ range from 7.5 to 11.4. Environ. Microbiol. 13, 3289-3309.

Jeon, C.O., Lim, J.M., Lee, J.M., Xu, L.H., Jiang, C.L. \& Kim, C.J. (2005). Reclassification of Bacillus haloalkaliphilus Fritze 1996 as Alkalibacillus haloalkaliphilus gen. nov., comb. nov. and the description of Alkalibacillus salilacus sp. nov., a novel halophilic bacterium isolated from a salt lake in China. Int. J. Syst. Evol. Microbiol. 55, 1891-1896.

Keller, J.M. et al. (2006). Equatorial and midlatitude distribution of chlorine measured by Mars Odyssey GRS. J. Geophys. Res. 111, E03S08.

Kounaves, S.P., Chaniotakis, N.A., Chevrier, V.F., Carrier, B.L., Folds, K. E., Hansen, V.M., McElhoney, K.M., O'Neil, G.D. \& Weber, A.W. (2014). Identification of the perchlorate parent salts at the Phoenix Mars landing site and possible implications. Icarus 232, 226-231.

Leshin, L.A. et al. \& MSL Science Team. (2013). Volatile, isotope, and organic analysis of martian fines with the Mars Curiosity rover. Science 341, 1238937/1-9.

LitchWeld, C.D. (2011). Potential for industrial products from the halophilic Archaea. J. Ind. Microbiol. Biotechnol. 38, 1635-1647.

Marion, G.M., Catling, D.C., Zahnle, K.J. \& Claire, M.W. (2010). Modeling aqueous perchlorate chemistries with applications to Mars. Icarus 207, 675-685.

Martín-Torres, F.J. et al. (2015). Transient liquid water and water activity at Gale crater on Mars. Nat. Geosci. 8, 357-361.

Maskow, T. \& Babel, W. (2003). Thermokinetic description of anaerobic growth of Halomonas halodenitrificans using a static microcalorimetric ampoule technique. J. Biotechnol. 101, 267-274.

McLennan, S.M. et al. (2005). Provenance and diagenesis of the evaporite-bearing Burns formation, Meridiani Planum, Mars. Earth Planet. Sci. Lett. 240, 95-121.

Melnyk, R.A., Clark, I.C., Liao, A. \& Coates, J.D. (2014). Transposon and deletion mutagenesis of genes involved in perchlorate reduction in Azospira suillum PS. mBio 5, e00769-e007613. 
Menezes, A.A., Cumbers, J., Hogan, J.A. \& Arkin, A.P. (2015). Towards synthetic biological approaches to resource utilization on space missions. J. R. Soc. Interface 12, 20140715.

Navarro-González, R., Vargas, E., de la Rosa, J., Raga, A.C. \& McKay, C.P. (2010). Reanalysis of the Viking results suggests perchlorate and organics at midlatitudes on Mars. J. Geophys. Res. 115, E12010.

Nozawa-Inoue, M., Jien, M., Hamilton, N.S., Stewart, V., Scow, K.M. \& Hristova, K.R. (2008). Quantitative detection of perchlorate-reducing bacteria by real-time PCR targeting the perchlorate reductase gene. Appl. Environ. Microbiol. 74, 1941-1944.

Ojha, L., Wilhelm, M.B., Murchie, S.L., McEwen, A.S., Wray, J.J., Hanley, J., Massé, M. \& Chojnacki, M. (2015). Spectral evidence for hydrated salts in recurring slope lineae on Mars. Nat. Geosci. 8, 829-832.

Oren, A., Elevi, B.R. \& Mana, L. (2014). Perchlorate and halophilic prokaryotes: implications for possible halophilic life on Mars. Extremophiles 18, 75-80.

Osterloo, M.M., Hamilton, V.E., Bandfield, J.L., Glotch, T.D., Baldridge, A.M., Christensen, P.R., Tornabene, L.L. \& Anderson, F.S. (2008). Chloride-bearing materials in the southern highlands of Mars. Science 319, 1651-1654.

Pieper, U., Kapadia, G., Mevarech, M. \& Herzberg, O. (1998). Structural features of halophilicity derived from the crystal structure of dihydrofolate reductase from the Dead Sea halophilic archaeon, Haloferax volcanii. Structure 6, 75-88.

Reed, C.J., Lewis, H., Trejo, E., Winston, V. \& Evilia, C. (2013). Protein adaptations in archaeal extremophiles. Archaea 2013, 373275.

Rey, M.W., Ramaiya, P., Nelson, B.A., Brody-Karpin, S.D., Zaretsky, E.J., Tang, M., de Leon, A.L., Xiang, H., Gusti, V., Clausen, I.G., Olsen, P.B., Rasmussen, M.D., Andersen, J.T., Jørgensen, P.L., Larsen, T.S., Sorokin, A., Bolotin, A., Lapidus, A., Galleron, N., Ehrlich, S.D. \& Berka, R.M. (2004). Complete genome sequence of the industrial bacterium Bacillus licheniformis and comparisons with closely related Bacillus species. Genome Biol. 5, R77.
Rothschild, J.L. (1990). Earth analogs for Martian life. Microbes in evaporites, a new model system for life on Mars. Icarus 88, 246-260.

Rothschild, L.J. (2016). Synthetic biology meets bioprinting: enabling technologies for humans on Mars (and Earth). Biochem. Soc. Trans 44, $1158-1164$.

Romano, I., Lama, L., Nicolaus, B., Gambacorta, A. \& Giordano, A. (2005). Alkalibacillus filiformis sp. nov., isolated from a mineral pool in Campania, Italy. Int. J. Syst. Evol. Microbiol. 55, 2395-2399.

Srinivasan, A. \& Viraraghavan, T. (2009). Perchlorate: health effects and technologies for its removal from water resources. Int. J. Environ. Res. Public Health 6, 1418-1442.

Tan, D., Wu, Q., Chen, J.C. \& Chen, G.Q. (2014). Engineering Halomonas TD01 for the low-cost production of polyhydroxyalkanoates. Metab. Eng. 26, 34- 47.

Tosca, N.J., Knoll, A.H. \& McLennan, S.M. (2008). Water activity and the challenge for life on early Mars. Science 320, 1204-1207.

Turner, S., Pryer, K.M., Miao, V.P. \& Palmer, J.D. (1999). Investigating deep phylogenetic relationships among cyanobacteria and plastids by small subunit rRNA sequence analysis. J. Eukaryot. Microbiol. 46, 327-338.

Vreeland, R.H., Litchfield, C.D., Martin, E.L. \& Elliot, E. (1980). Halomonas elongata, a new genus and species of extremely salt-tolerant bacteria. Int. J. Syst. Evol. Microbiol. 30, 485-495.

Wang, Y., Wu, Y.H., Wang, C.S., Xu, X.W., Oren, A., Zhu, X.F. \& Wu, M. (2008). Halomonas salifodinae sp. nov., a halophilic bacterium isolated from a salt mine in China. Int. J. Syst. Evol. Microbiol. 58, 2855-2858.

Yue, H., Ling, C., Yang, T., Chen, X., Chen, Y., Deng, H., Wu, Q., Chen, J. \& Chen, G. (2014). A seawater-based open and continuous process for polyhydroxyalkanoates production by recombinant Halomonas campaniensis LS21 grown in mixed substrates. Biotechnol. Biofuels 7, 108.

Zehr, J.P., Harvey, R.W., Oremland, R.S., Cloern, J.E., George, L.H. \& Lane, J.L. (1987). Big Soda Lake (Nevada). 1. Pelagic bacterial heterotrophy and biomass. Limnol. Oceanogr. 32, 781-793. 